\title{
Perforation of a Nonobstructing Gastro-Oesopha- geal Carcinoma by Oblique-Viewing Endoscopic Ultrasound Videoscope: A Need for a Safe Technique
}

Endoscopic ultrasound (EUS) is the gold standard investigation for assessing the $\mathrm{T}$ and $\mathrm{N}$ stages of oesophageal carcinoma [1]. The standard EUS probe is mounted on a side-viewing endoscope, making intubation of the oesophagus a more complex procedure. The recently developed EUS video scope, although oblique-viewing, provides a view almost as good as the standard endviewing endoscope. This apparent improvement in design can mislead the endoscopist and may increase the risk of perforation. We report a case of perforation of a nonobstructing oesophageal cancer with the new EUS scope.

Endoscopic assessment of a 68-year-old man revealed a soft fleshy tumour of the oesophagogastric junction, which was easily traversible. Subsequent intubation of the oesophagus with the oblique-viewing video EUS scope provided an adequate view of the lumen. Figure $\mathbf{1}$ a illustrates this in a similar case. As the EUS scope was negotiated through the tumour, the tip of the endoscope which points laterally to provide a view of the lumen as shown in Figure $\mathbf{1} \mathbf{b}$, penetrated the full thickness of the friable tumour to come
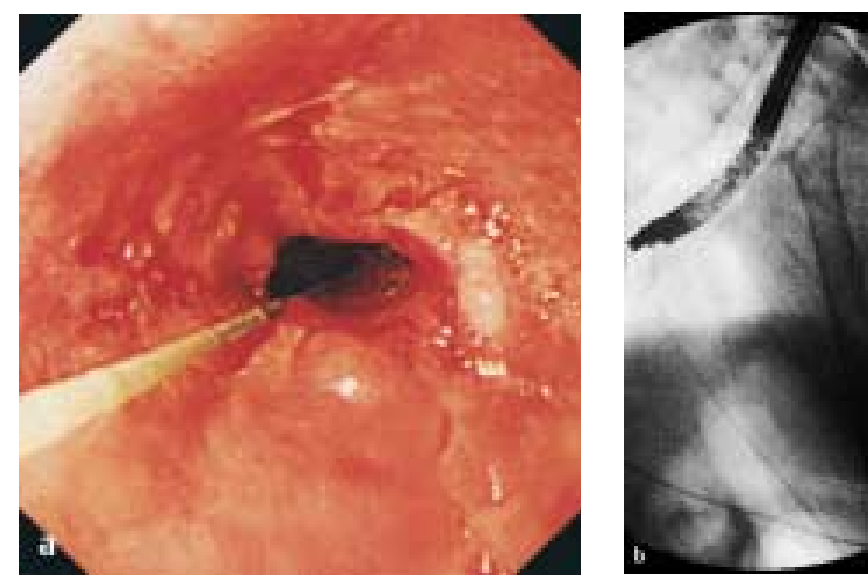

Figure 1 a Image of the lumen of the oesophagus taken with the video endoscopic ultrasonography (EUS) scope, showing a guide wire passing through a circumferential carcinoma of the lower oesophagus. b This radiograph was taken concomitantly, as the EUS scope revealed the view in a. This illustrates that in order to obtain such as view of the lumen the tip of the video EUS scope must point laterally into one wall of the oesophagus. Further advancement in this position may result in a perforation. to lie freely in the peritoneal cavity. The patient underwent emergency surgery and made a full recovery. Since this serious complication occurred we have changed our technique for intubation with the EUS scope.

After the initial endoscopic assessment (the oesophageal tumour is dilated if required, under image intensification), a floppy guide wire is placed in the gastric antrum. The EUS scope is then introduced into the oesophagus and negotiated through the tumour with the tip of the video scope closely following the path of the guide wire under the guidance of the image intensifier (Figure 2). We employ this technique for all our EUS staging of oesophageal carcinoma whether or not dilation is required because although the incidence of perforation is low [2], when it does occur, it can often be fatal and converts what might be a potentially curable condition to one in which only palliation is possible. Hence we feel that any technique which minimizes the risk of this infrequent but potentially serious complication is worth employing. We have found this technique safe and have not encountered any EUS-related oesophageal perforations in approximately 350 cases since the one reported here.

M. V. Chandrashekar ${ }^{1}$, D. L. Richardson ${ }^{2}$, S. Preston ${ }^{1}$, D. Karat ${ }^{1}$, S. M. Griffin ${ }^{1}$

${ }^{1}$ Northern Oesophago-Gastric Cancer Unit, Royal Victoria Infirmary, Newcastle-upon-Tyne, UK

${ }^{2}$ Department of Radiology, Royal Victoria Infirmary, Newcastle-upon-Tyne, UK

\section{References}

${ }^{1}$ Dittler HJ, Siewart JR. Role of endoscopic ultrasonography in oesophageal carcinoma. Endoscopy 1993; 25: 156-161

${ }^{2}$ Cotton P, Williams C. Practical gastrointestinal endoscopy; 4th edn.: 75

Corresponding Author

\section{S. M. Griffin, M.D.}

North. Oesophago-Gastric Cancer Unit Ward 36 Office, Royal Victoria Infirmary Queen Victoria Road

Newcastle-upon-Tyne NE1 4PL, UK

Fax: $\quad$ + 44-191-2820237

E-mail: s.m.grfffm@ncl.ac.uk

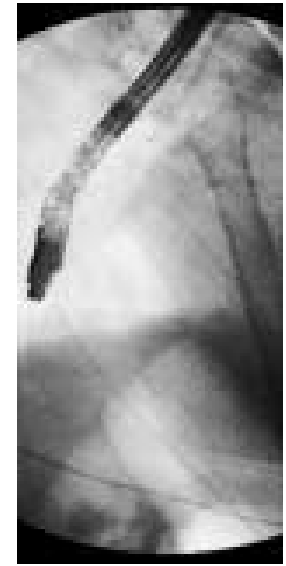

Figure 2 The radiograph shows the guide wire in the lumen of the oesophagus with the tip of the EUS scope following the guide wire through the tumour, minimizing the risk of perforation. 